\title{
miR-133b acts as a tumor suppressor and negatively regulates EMP2 in glioma
}

\author{
Q. ZHANG ${ }^{1,2}$, X. FAN ${ }^{3}$, B. XU ${ }^{2}$, Q. PANG ${ }^{1}$, L. TENG ${ }^{1, *}$ \\ ${ }^{1}$ Department of Neurosurgery, Shandong Provincial Hospital Affiliated to Shandong University, Jinan 250000, China; ${ }^{2}$ Department of \\ Neurosurgery, Binzhou People's Hospital, Binzhou 256600, China; ${ }^{3}$ Department of Cadre Health Care, Binzhou People's Hospital, Binzhou \\ 256600, China
}

*Correspondence: Tenglz229@126.com

Received May 10, 2017 / Accepted September 1, 2017

\begin{abstract}
In recent years, the incidence of neuroglioma (glioma) has trended towards a younger age-group. Gene therapy has been widely implemented and a growing number of microRNAs associated with glioma have been identified., Herein, we detected the expression of micro RNA - miR-133b - in glioma by qPCR and also its effect on cell viability, survival and apoptosis of in vitro U87 and A172 cells. The binding effect of miR-133b on epithelial membrane protein-2 (EMP2) was verified and we then investigated the effect of EMP2 on in vitro glioma cells and tested the expression of apoptosis related factors after administration of altered miR-133b and EMP2 expressions. We found that miR-133b was down-regulated in glioma compared to adjacent non-tumorous tissue and also that its over-expression inhibits cell viability and survival and enhances apoptosis in the U87 and A-172 cells. Moreover, miR-133b effectively binds to EMP2, down-regulates its expression and negates its normal function. EMP2 normally promotes cell apoptosis and reduces cell viability and survival while miR-133b over-expression regulates the expression of apoptotic-associated protein and activates the apoptotic pathway, thus counteracting EMP2 regulation of opposite expression effects. Further, miR-133b can be considered a tumor suppressor because of its low expression and effects on cell apoptosis via down-regulating EMP2 expression and activating the apoptotic cell pathway in glioma. EMP2 is a risk factor for glioma, and miR-133b should prove a potential target for glioma clinical prevention and treatment.
\end{abstract}

Key words: glioma, microRNA-133b, epithelial membrane protein-2, apoptosis, tumor suppressor

Neuroglioma (glioma) is the most common intracranial primary malignant tumor seen in clinical neurosurgery, and it accounts for $40-50 \%$ of central nervous system tumors [1]. Gliomas occur anywhere in the central nervous system, but primarily in brain glial tissue where it has high recurrence and mortality and low cure rate [2]. This complex neuroglia tumor involves dysregulation of many biological pathways at multiple levels [3], and it has been demonstrated that glioma development is related to oncogene over-expression and inactivation of tumor suppressor genes; including Dikkopf-1, Hox homeotic genes, Leucine-rich repeats and the 1 (LRIG1) gene immunoglobulin-like domains [1, 4]. Unfortunately, glioma's precise molecular mechanisms still remain unknown.

MicroRNAs (miRNAs) have been suggested to play a vital role in tumor initiation and progression by negatively regulating oncogenes and tumor suppressors [5]. A growing number of miRNAs have been investigated for their effects on tumor cells and their association with tumor malignancy; including glioma [6]. miR-133b which was initially considered a muscle-specific miRNA has been demonstrated to have abnormal expression in several cancer cell lines and tumors [7-9]. Although many miRNAs have been implicated in glioma carcinogenesis, including miR-21, miR-128 and miR-26a [10-12], the role of miR-133b in mediating glioma cells has not previously been elucidated.

This study is designed to examine the expression of miR-133b in glioma tissue and its biological effects on glioma cells. We measured the effects of miR-133b expression on cell viability, survival and apoptosis in glioblastoma cell lines U87 and A172. Epithelial membrane protein-2 (EMP2) is a predicating target of miR-133b, so the regulation of EMP2 expression by miR-133b and the effects of EMP2 on glioma cells growth were also assessed in vitro. Finally, we investigated the possible mechanism of miR-133b in regulating apoptosis by validating the effects of EMP2 expression on apoptosis related factors in vitro. 


\section{Patients and methods}

Ethical statement and tissue samples. Paired glioma and adjacent non-tumorous tissues were obtained from 5 patients who had resection in the local hospital between January 2013 and December 2015, and none of these patients had received adjuvant therapy before the operation. The study was approved by the ethical board of our hospital and all samples were obtained from patients who signed informed consent approving the use of their tissues for research purpose after their operation. Fresh tissues were sampled directly in the operation and then immediately frozen in liquid nitrogen and used in the miRNA micro-array experiments.

Cell culture. Human cell line U87, most likely glioblastoma, and human glioblastoma cell line A172 were obtained from American Type Culture Collection (HTB-14 ${ }^{\mathrm{Tx}}$, CRL-1620 $0^{\text {rs }}$; ATCC, Manassas, VA, USA) and kept in our laboratory. Cells were cultured for this study in Dulbecco's Modified Eagle's Medium (DMEM, Gibco BRL, Gaithersburg, MD) culture medium containing 10\% fetal-calf-serum (Gibco, Grand Island, NY, USA). They were then stored in an incubator containing $5 \% \mathrm{CO}_{2}$, at $37^{\circ} \mathrm{C}$.

RNA extraction and real-time quantitative PCR (qPCR). Total tissue cell RNA was extracted by Trizol regent (Invitrogen Life Technologies, Carlsbad, CA, USA) and RNA quantity was determined by electrophoresis. For cDNA synthesis, total RNA was reverse-transcribed by Transcriptor First Stand Synthesis Kit (Roche, Indianapolis, IN, USA) according to the manufacturer's instructions. The qPCR was performed by master SYBRH Premix Ex Taq ${ }^{\mathrm{TM}}$ (TaKaRa, Biotech Co., Ltd, Dalia, China) and each sample was carried out in triplicate on the ABI PRISM 7500 Real-time PCR System (Applied Biosystems, Foster City, CA, USA). Data was analyzed by the classic $2^{-\Delta \Delta \mathrm{Ct}}$ method, and normalized to GAPDH or U6 snRNA expression in each sample [13].

Cell transfection. The cells were plated in $60 \mathrm{~mm}$ dishes and cultured for 24 hours and then transfected with miR-133b mimic, inhibitor, mimic control and inhibitor control (GenePharma, shanghai, China). Recombinant vector pcDNA3.1 (Thermo Fisher Scientific, Waltham, MA, USA) with the full length of EMP2 coding sequence (EMP2), empty vector, small interfering RNA (siRNA) against EMP2 (EMP2 siRNA), or non-targeting siRNA (si-control) (GenePharma) were transfected into U87 and A172 cells. All transfections were performed by Lipofectamine 3000 (Invitrogen) according to the manufacturer's instructions. The treated cells were observed six hours after transfection, and serum free medium was changed to complete culture medium. The cells were then collected for further analysis after 48-hour transfection.

Cell viability assay. Cells were seeded in 96-well plates at $5 \times 10^{3}$ cells/well and then transfected with miR-133b mimic, mimic control, miR-133b inhibitor or inhibitor control and cultured for 24-72 hours. After incubation with 3-(4, 5-dimethylthiazolyl-2)-2, 5-diphenyltetrazolium bromide
(MTT, Sigma-Aldrich St. Louis, MO, USA), viable cells were lysed in $150 \mathrm{ml}$ of $100 \%$ dimethyl sulfoxide (DMSO, SigmaAldrich) and UV-visible absorbance was read at $490 \mathrm{~nm}$ by 680 microplate enzyme-linked immunosorbent assay (ELISA) reader (Bio-Rad, Hemel Hempsted, UK).

Cell survival assay. Treated cells were seeded into a new $60 \mathrm{~mm}$ cell culture dish $\left(1 \times 10^{3}\right.$ cells/well $)$ and incubated for 9 days to obtain the survival capability of transfected cells, Culture medium in each well was removed and $1 \mathrm{ml}$ Clonogenic Reagent (50\% Ethanol, 0.25\% 1,9-dimethyl-methylene blue, Sigma-Aldrich) was added, and kept at room temperature for 45 minutes. Cells were then washed and blue-stained colonies were counted.

Detection of cell early apoptosis. Triplicate quantitation of apoptotic cells was performed by Annexin V-FITC/PI apoptosis kit (BioVision, Milpitas, CA, USA). After transfection, U87 and A172 cells were resuspended in $500 \mu$ l binding buffer, and then dyed with $5 \mu$ l Annexin V-FITC and PI. After 10 minutes incubation in the dark at room temperature, the early apoptotic cells were distinguished by flow cytometer (BD Biosciences, San Jose, CA, USA).

miRNA targets prediction and dual luciferase activity assay. The putative miR-133b targets in glioma cells were predicted using TargetScan, and EMP2 was proposed according to miRBase and PicTar algorithms. The EMP2 3'UTR that contains the putative miR-133b site was cloned into pGL2-basic vector as pmirGL2/EMP2-UTR. The mutated EMP2 3'UTR was then cloned into Pgl2-basic vector as pmirGL2/EMP2-UTR mutant. The cells for this test were divided into the following groups according to different transfections: EMP2 3'UTR (pmirGL2/EMP2-UTR)+ miR133b mimic control; EMP2 3'UTR + miR133b mimic; EMP2 3'UTR + miR133b inhibitor control; EMP2 3'UTR + miR133b inhibitor;EMP2 3'UTRMut (pmirGL2/EMP2-UTR mutant)+ miR133b mimic control; EMP2 3'UTR Mut + miR133b mimic; EMP2 3'UTR Mut + miR133b inhibitor control and EMP2 3'UTR Mut + miR133b inhibitor.

In brief, triplicate runs of cells were seeded in 96-well plates and cultured for 24 hours and then co-transfected with different contents using Lipofectamine 2000 (Invitrogen). Firefly and Renilla luciferase activity were measured using the Dual-Luciferase reporter system (Promega, Madison, WI, USA) after the 48 hour transfection [14].

Western blotting. Western blotting determined EMP2 and apoptosis-related factor expression. The cellular proteins were extracted after transfection by lysis buffer (Beyotime Biotechnology, Nantong, Jiangsu, China) and samples were resolved on an $8 \%$ sodium dodecyl sulfate (SDS)-denatured polyacrylamide gel and blots were then transferred onto a nitrocellulose membrane. Membranes were incubated with 1:1,000 primary antibodies overnight at $4^{\circ} \mathrm{C}$. The primary antibodies used were as follows; EMP2 (sc101949; Santa Cruz, CA, USA); cleaved-caspase-3 (sc271759; Santa Cruz); B-cell lymphoma (Bcl-2, sc509; Santa Cruz); Bcl-2-associated X (Bax, sc20288; Santa Cruz); c-Jun N-terminal kinase (JNK, 
sc7345; Santa Cruz); phosphorylated JNK (p-JNK, sc6254; Santa Cruz); p38(sc7149; Santa Cruz); phosphorylated p38(p-p38, sc166182; Santa Cruz) and GAPDH (sc365062; Santa Cruz).

The membranes were washed and incubated for 1 hour at room temperature with horseradish peroxidase (HRP)conjugated secondary antibody. The band immunoreactivity was developed by enhanced chemi-luminescent film and band intensities were quantified by LabWorks image acquisition and analysis software (UVP LLC, Upland, CA, USA).

Statistical analysis. Data was analyzed by GraphPad Prism 6 software (GraphPad Software, San Diego, CA, USA)I and presented as mean \pm standard deviation (SD) The two-tailed Student's t-test evaluated the significance of differences between the two groups, with one-way analysis of variance (ANOVA) evaluating the significance of differences in mean values within and between multiple groups at $\mathrm{p}<0.05$ significance.

\section{Results}

miR-133b was down-regulated in glioma tissues. The miR-133b expression in tumorous and adjacent non-tumorous tissues was detected by qPCR, and Figure 1 highlights that miR-133b expression was lower in glioma tissue than in non-tumor tissues $(\mathrm{p}<0.001)$. The expression level of miR-133b negatively correlated with glioma.

The expression level of miR-133b was significantly changed after transfection with both mimic and inhibitor. We screened cells with high transfection efficiency, and miR-133b expression in U87 and A172 cells was detected by qPCR after transfection with miR-133b mimic or inhibitor. Results showed that miR-133b was efficiently over-expressed in U87 cells in the miR-133b mimic group and inhibited in the miR-133b inhibitor group (Figure $2 \mathrm{~A}$ ), and this effect was also noted in A172 cells (Figure $2 \mathrm{~B})(\mathrm{p}<0.01)$. This indicated

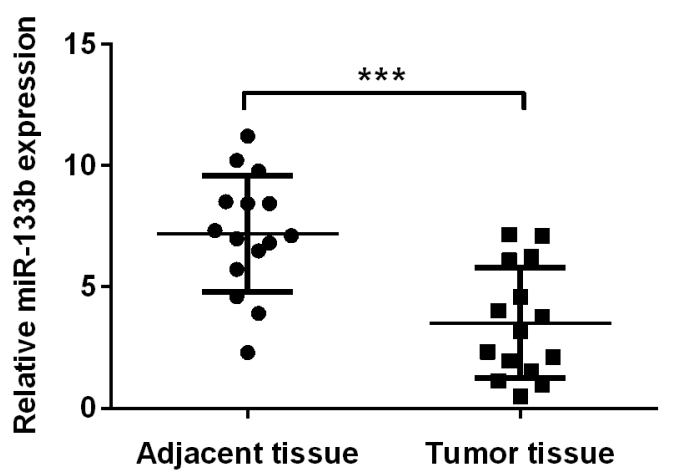

Figure 1. miR-133b expression levels are down-regulated in glioma. The expression levels of miR-133b in glioma tissues and adjacent non-tumor tissue samples were measured by $q$ PCR. miR-133b, microRNA-133b; qPCR, quantitative polymerase chain reaction; ${ }^{* *}, \mathrm{p}<0.001$. that these transfections with miR-133b mimic or inhibitor were effective in U87 and A172 cells.

Over-expression of miR-133b inhibited U87 and A172 cell viability and survival. The results of MTT assay in Figures $2 \mathrm{C}$ and $\mathrm{D}$ reveal that miR-133b over-expression decreased cell viability in both U87 and A172 cells $(\mathrm{p}<0.01)$, while cell viability was increased after transfection in miR-133b inhibitor treated groups $(\mathrm{p}<0.05)$. This trend was also found in survival assay results (Figure $2 \mathrm{E}$ and $2 \mathrm{~F}$ ) where the U87 and A172 cell survival rate in miR-133b mimictransfected was lower than in the mimic control group $(\mathrm{p}<0.05)$. Moreover, the survival rates of these two cells were increased in the miR-133b inhibitor groups, $(p<0.05)$, and this infers that $\mathrm{miR}-133 \mathrm{~b}$ expression negatively corrrelates to cell viability and survival in both U87 and A172 cells.

Over-expression of miR-133b promotes early U87 and A172 cell apoptosis. Our results in Figures $2 \mathrm{G}$ and $\mathrm{H}$ confirm early U87 and A172 cell apoptosis after transfection. The apoptotic rate of miR-133b mimic-transfected cells was significantly decreased $(\mathrm{p}<0.001)$ compared to the miR-133b mimic control group. In contrast, the apoptotic rates of these cells were dramatically increased in the miR-133b inhibitor transfection groups, $(\mathrm{p}<0.01$ or $\mathrm{p}<0.001)$, and this suggests that miR-133b over-expression promotes U87 and A172 cell apoptosis.

miR-133b negatively regulates EMP2 expression via directly targeting it in both U87 and A172 cells. The TargetScan prediction in Figure 3A identifies miR-133b's potential EMP2-3'UTR binding sites. We synthesized the double stranded sequence of EMP2 containing the miR-133b binding sites and assessed the binding effect of miR-133b on EMP2 in U87 and A172 cells. The results in Figure 3 B-E establish that fluorescence signal intensity was significantly decreased after EMP2 3'UTR and miR-133b mimic co-transfection compared to EMP2 3'UTR transfection alone $(\mathrm{p}<0.01)$; but the opposite trend was seen in the miR-133b inhibitor groups, $(\mathrm{p}<0.01)$ The fact that there were no significant differences in the EMPe Mut treated group indicates that miR-133b directly binds to EMP2 3'UTR, and that EMP2 is miR-133b's direct target in the U87 and A172 cells.

We also assessed the effect of miR-133b on EMP2 expression, and the results in Figure 4 indicate that miR-133b overexpression in U87 and A172 cells significantly reduced the EMP2 mRNA expression level $(\mathrm{p}<0.01)$ and that miR-133b inhibition improved the EMP2 mRNA expression level $(\mathrm{p}<0.01)$ (Figure $4 \mathrm{~A}$ and $4 \mathrm{~B})$. Further, Figures $4 \mathrm{C}$ and $\mathrm{D}$ show that the EMP2 protein expression level was lower in the miR-133b mimic treated group than in the miR-133b mimic control, and this increased after miR-133b inhibitor transfection. The expression changes in EMP2 therefore indicate that miR-133b negatively regulates EMP2 expression in U87 and A172 cells via targeting the EMP2 promoter.

EMP2 is up-regulated in glioma tissues and is positively related to cell viability and survival. After confirming EMP2 as a miR-133b target gene and the effect of miR-133b on U87 
A

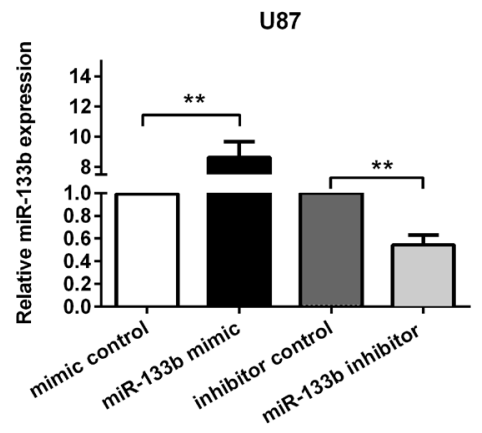

C

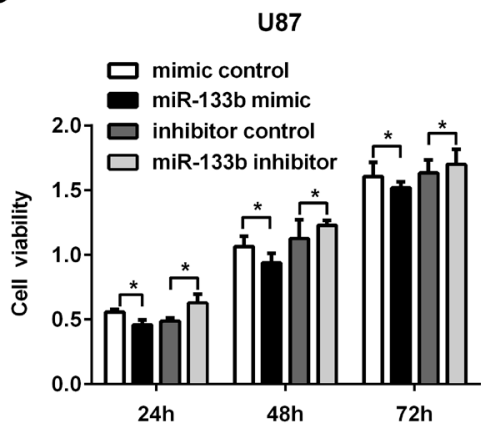

E

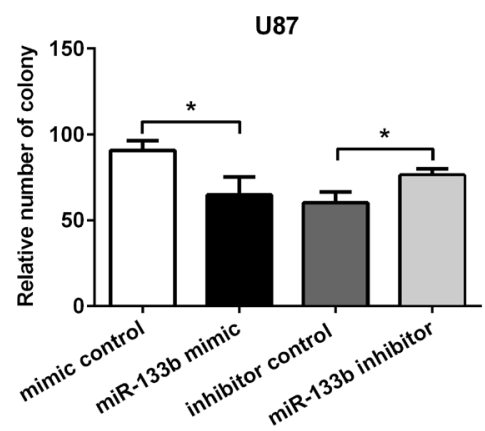

G

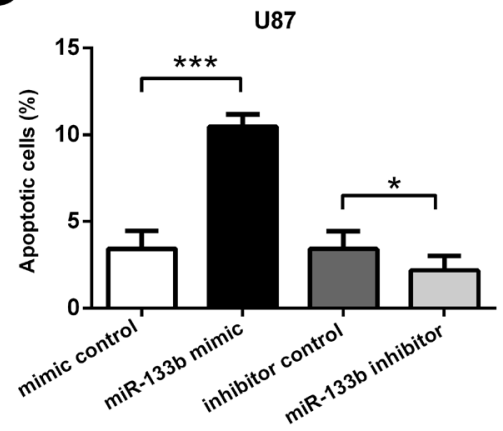

B

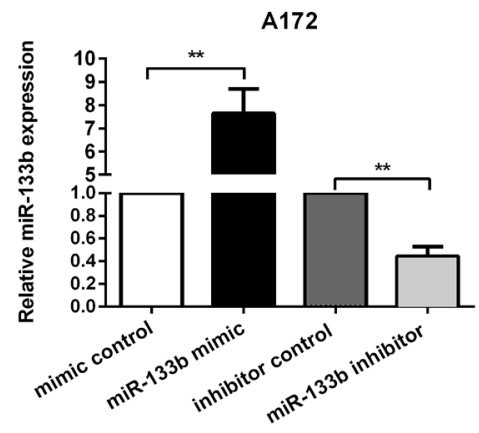

D

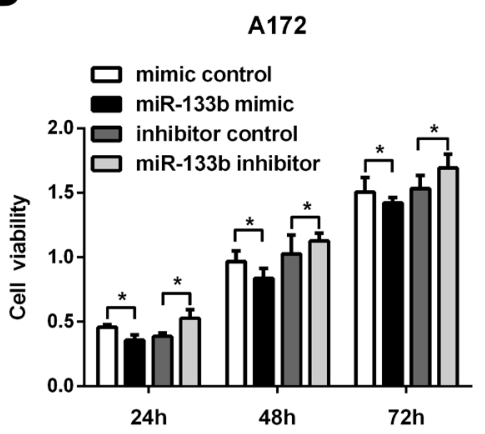

$\mathbf{F}$

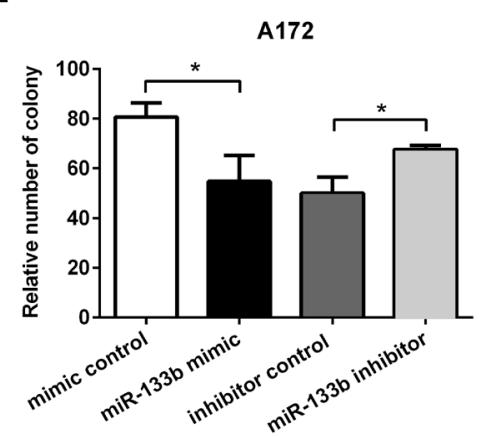

H

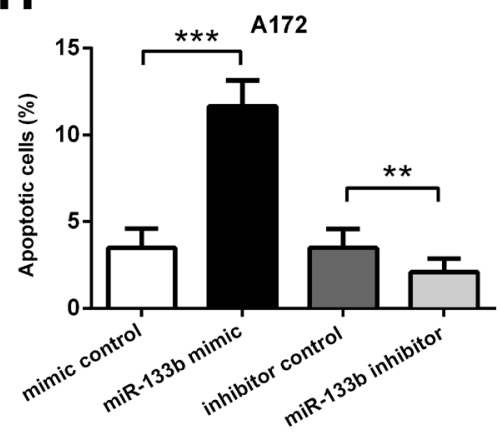

Figure 2. miR-133b over-expression suppressed cells viability and survival and increased apoptosis of glioma cells in vitro. The miR-133b mimic, inhibitor, mimic control or inhibitor control was transfected into U87 and A172 cells, respectively. (A and B) The transfection efficiency in U87 and A172 cells was tested by qPCR. (C and D) The transfected U87 and A172 cells were collected and cultured for 24-72 h, and cell viability was determined by MTT assay. (E and F) The proliferation of transfected U87 and A172 cells were measured by colony formation assay. (G and H) The apoptotic cells rate of U87 and A172 cells were detected by flow cytometry. miR-133b, microRNA-133b; qPCR, quantitative polymerase chain reaction; MTT, 3-(4, 5-dimethyl-2-thiazolyl)-2, 5-diphenyltetrazolium bromide; ${ }^{*}, \mathrm{p}<0.05$; ${ }^{* *}, \mathrm{p}<0.01 ;{ }^{* * *}, \mathrm{p}<0.001$. 
A

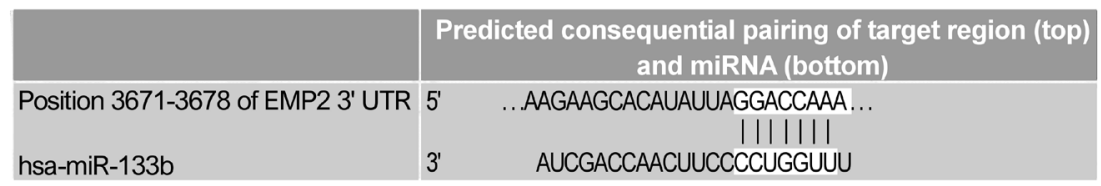

B

D

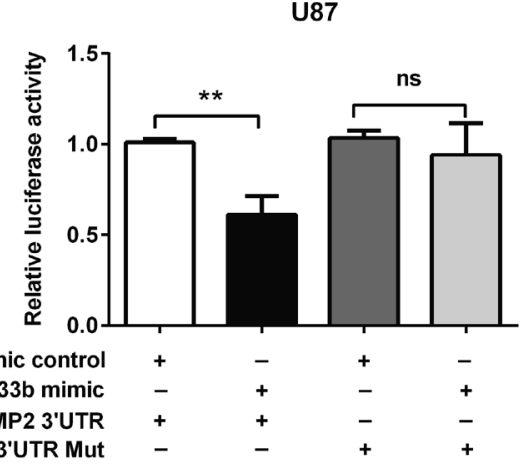

D

A172

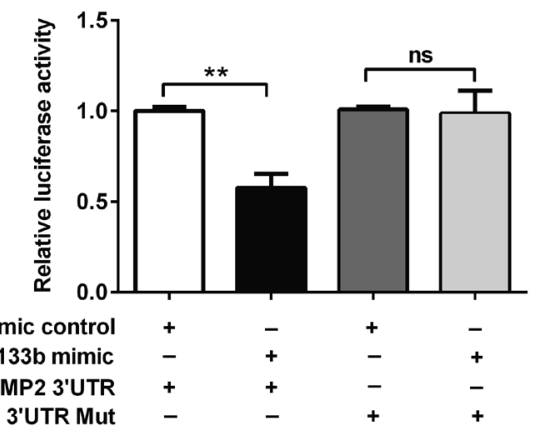

C

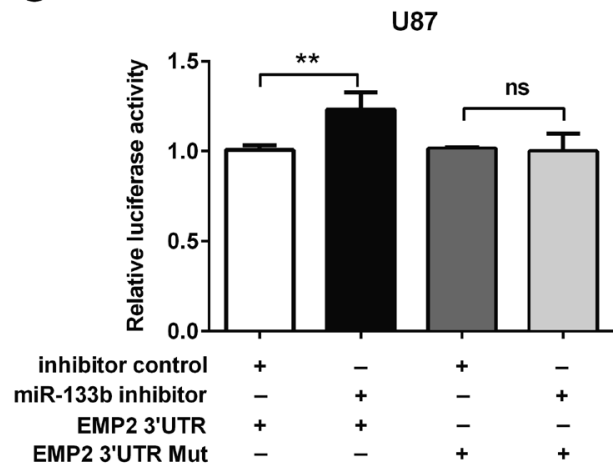

E

A172

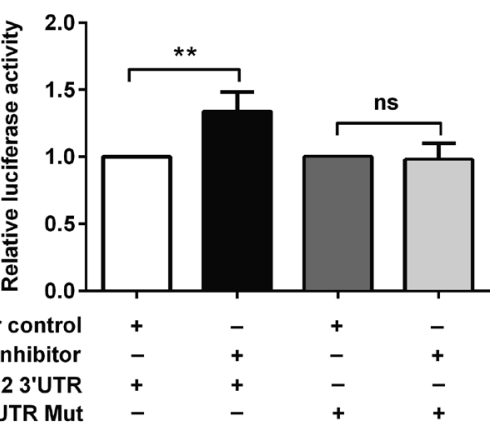

Figure 3. EMP2 was a direct target of miR-133b. (A) EMP2 3'UTR region showing potential miR-133b alignment. (B and C) The targeting effect of miR-133b on EMP2 in U87 cells was detected by luciferase reporter assay. (D and E) Luciferase reporter assay showed the effect of miR-133b on EMP2 in A172 cells. miR-133b, microRNA-133b; EMP2, epithelial membrane protein-2; 3'-UTR, 3'-untranslated regions; ns, no significant influence; **, $\mathrm{p}<\mathbf{0 . 0 1}$.

and $\mathrm{A} 172$ cells, we performed preliminary research on the role of EMP2 in glioma. Figure 5A shows that EMP2 expression in glioma is higher than in adjacent non-tumorous tissues $(p<0.001)$. We also confirmed that EMP2 can be efficiently over-expressed or inhibited by exogenous expression and also by EMP2 siRNA silencing; as shown in Figure 5B.

We then examined EMP2's effect on U87 and A172 cells and established that exogenous EMP2 expression enhanced cell viability (Figures 5C, D) and also survival (Figures 5E, F). In contrast, EMP2 siRNA administration inhibited these cells' viability and survival $(\mathrm{p}<0.05$ and $\mathrm{p}<0.01)$, and the apoptosis assay revealed that the relative apoptosis rate of U87 and A172 cells was reduced after EMP2 over-expression. Figures5G and $\mathrm{H}$ show the opposite effect in the EMP2
siRNA group $(\mathrm{p}<0.001)$ and this confirms that up-regulated EMP2 presents a risk factor for glioma cells.

Inhibition of miR-133b and EMP2 expression promotes cell viability and survival and inhibits apoptosis in U87 and $\mathbf{A 1 7 2}$ cells. We further analyzed cell viability, survival and apoptosis rates in miR-133b inhibition, with and without the EMP2 inhibited expression, in order to confirm the relationship between miR-133b, EMP2 expression, cell viability, survival and apoptosis. Figures $6 \mathrm{~A}$ and $\mathrm{B}$ establish that miR-133b inhibition increased cell viability in both U87 and A172 cells $(\mathrm{p}<0.05)$, and in contrast, combined inhibited expression of miR-133b and EMP2 decreased cell viability $(\mathrm{p}<0.05)$. This same trend was supported by our survival assay results (Figures 6C and D). While the U87 and A172 
A

U87

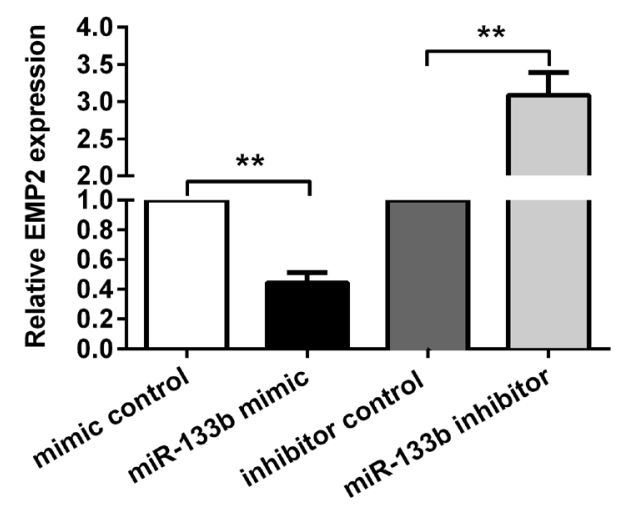

C

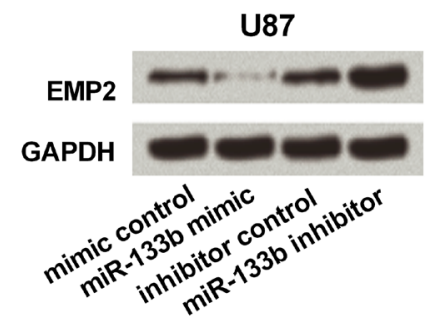

B

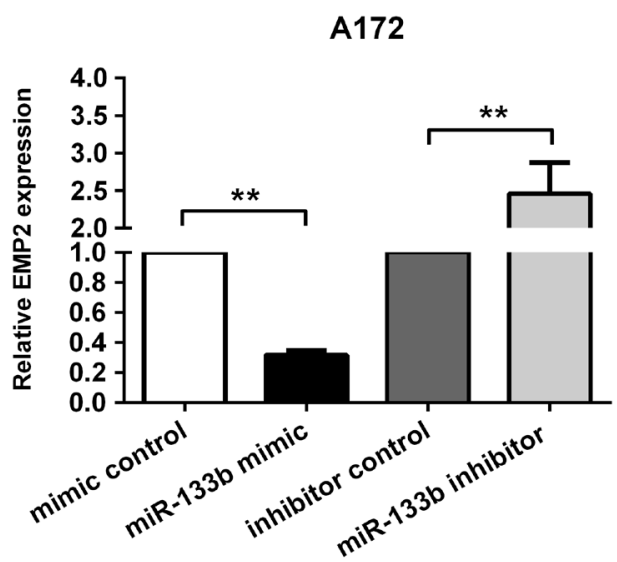

D

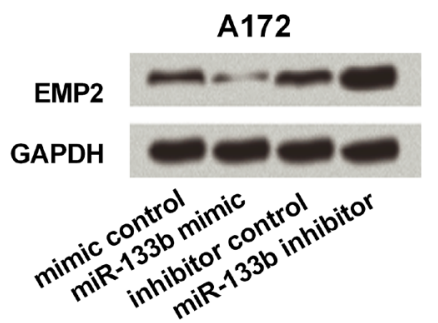

Figure 4. miR-133b negative regulated the expression of EMP2. (A and B) The mRNA expression levels of EMP2 in transfected U87 and A172 cells were detected. U6 acted as an internal control. (C and D) The protein expression levels of EMP2 in miR-133b mimic or inhibitor treated U87 and A172 cells. GAPDH acted as an internal control. miR-133b, microRNA-133b; EMP2, epithelial membrane protein-2; ${ }^{\star \star}, \mathrm{p}<0.01$.

cell survival rates in miR-133b inhibitor and EMP2 siRNA transfected groups were lower than in the miR-133b inhibitor and si-control group $(\mathrm{p}<0.05)$, their survival rates increased in the miR-133b inhibitor groups, $(\mathrm{p}<0.05)$.

Figures $6 \mathrm{E}$ and $\mathrm{F}$ apoptosis assay results highlight that the apoptosis rates of miR-133b inhibitor transfected cells (U87 and A172 cells) were significantly decreased $(\mathrm{p}<0.05)$ compared to the miR-133b inhibitor control group, and also that apoptosis rates in both cells were dramatically increased in the miR-133b inhibitor and EMP2 siRNA transfection groups, $(\mathrm{p}<0.05)$. These results not only suggest that miR-133b inhibition promoted cell viability and survival and inhibited cell apoptosis in the U87 and A172 cells, but also that inhibited miR-133b and EMP2 expression did not further promote these cells' viability and survival or inhibit cell apoptosis.

Possible miR-133b pathway in the regulation of glioma cell apoptosis. Cleaved-caspase-3, Bcl-2, Bax, p-38 and JNK protein expression levels were detected in order to study whether miR-133b regulation of EMP2 was involved in EMP2-mediated signal transduction of glioma cell apoptosis. Figure 7 confirms their protein expression increase after miR-133b mimic transfection and their decrease after miR-133b inhibitor transfection. In contrast, $\mathrm{Bcl}-2$ expression and EMP2 had the opposite effects on these proteins' expression. Finally, we consider that miR-133b regulates apoptosisrelated signaling pathways through targeting EMP2 because it directly targets EMP2 and negatively regulates its expression.

\section{Discussion}

Glioma is a common infiltrative intracranial tumor lacking clear boundaries with normal brain tissue. Therefore, it is extremely difficult to be completely removed $[15,16]$. Moreover, gliomas are relatively insensitive to both radiotherapy and chemotherapy and they relapse very easily [17]. Curative effects for glioma are also not ideal because of the relatively impermeable blood-brain barrier [18] and glioma therefore remains one of the worst prognostic tumors. It develops from genetic alterations accumulated throughout tumor progression [19-21], and understanding the molecular mechanisms involved is crucial for better prediction of glioma patient outcome and therapy response. This is especially true for mechanisms associated with cellular migration and invasion.

The molecular pathogenesis mechanism of glioma has therefore attracted considerable attention [22]. miRNAs are 
A

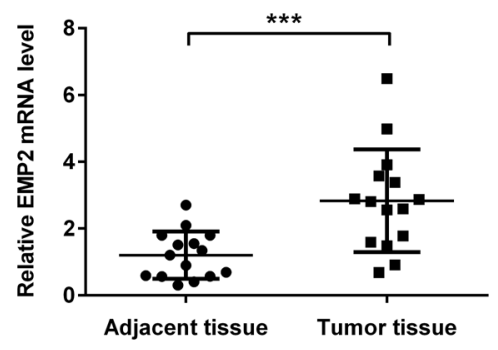

C

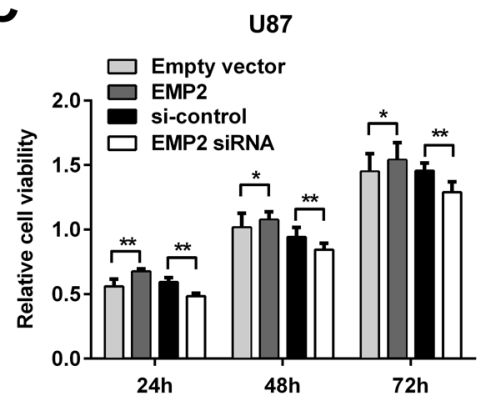

E

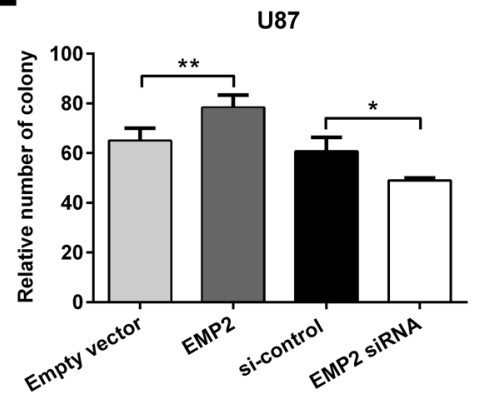

G

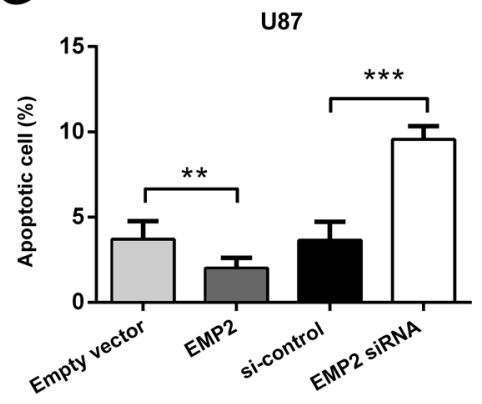

B

U87

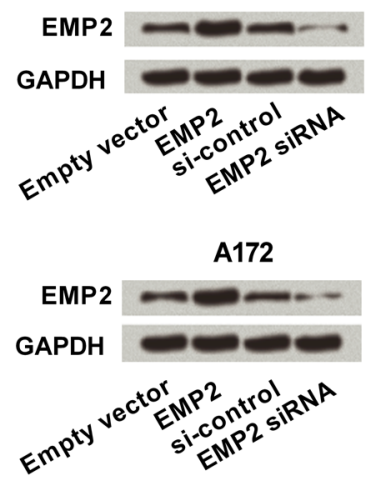

D

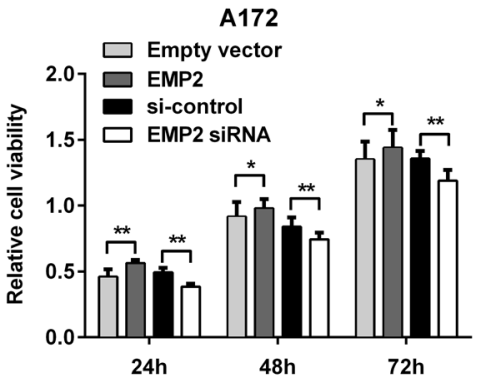

F

A172

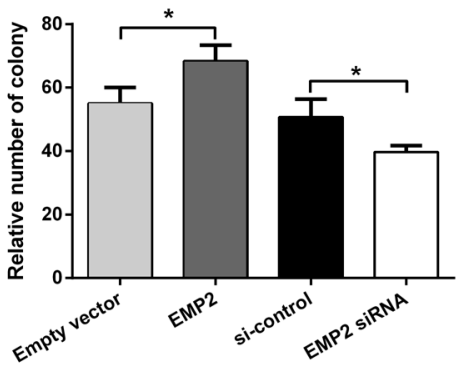

H

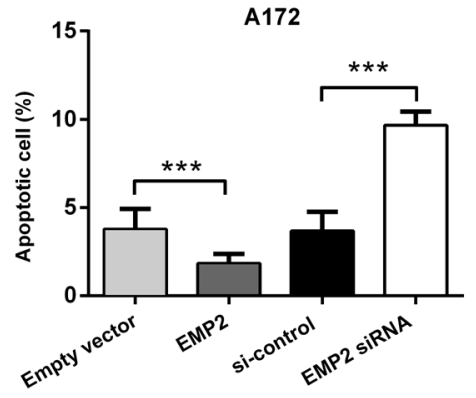

Figure 5. Overexpression of EMP2 promoted cell viability and survival, and inhibited apoptosis in U87 and A172 cells. (A) The expression of EMP2 detected by $\mathrm{qPCR}$ was upregulated in glioma. (B) The transfection efficiency of recombined vector pcDNA3-EMP2 or EMP2 siRNA was tested by Western blotting. (C and D) EMP2-transfected cells viability of U87 and A172 cells for 24-72 h were determined by MTT assay. (E and F) The cell survival of EMP2-transfected U87 and A172 cells were measured by colony formation assay. (G and H) The apoptotic cells rates of U87 and A172 cells were detected by flow cytometry. EMP2, epithelial membrane protein-2; qPCR, quantitative polymerase chain reaction; siRNA, small interfering RNA; MTT, 3-(4, 5-dimethyl-2-thiazolyl)-2, 5-diphenyltetrazolium bromide; ${ }^{\star}, \mathrm{p}<0.05$; ${ }^{\star *}, \mathrm{p}<0.01$; ${ }^{\star * \star}, \mathrm{p}<0.001$. 
A

U87

Inhibitor control

miR-133b inhibitor

miR-133b inhibitor+si-control

miR-133b inhibitor+EMP2 siRNA

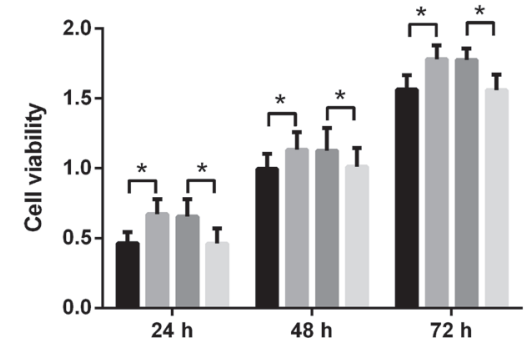

C
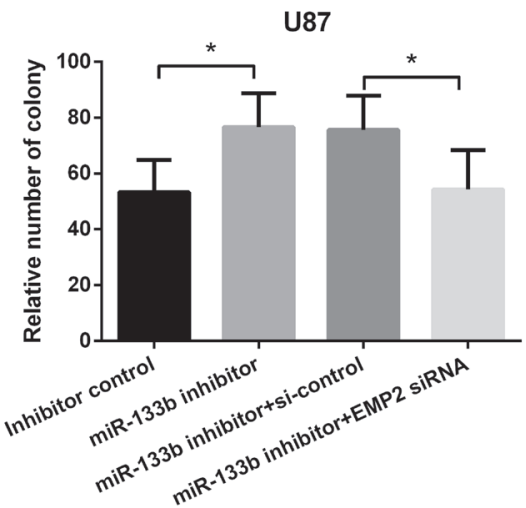

E

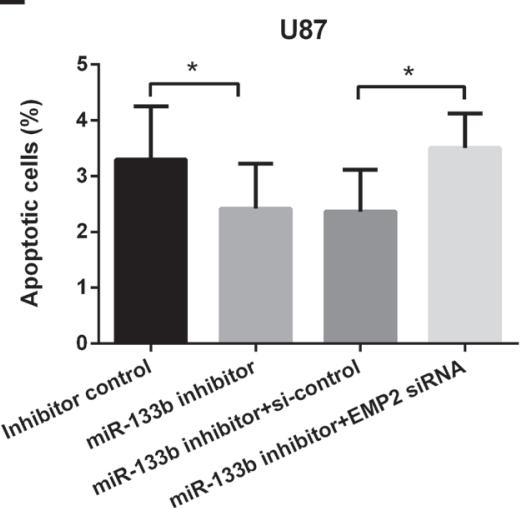

B

Inhibitor control

miR-133b inhibitor

miR-133b inhibitor+si-control

miR-133b inhibitor+EMP2 siRNA

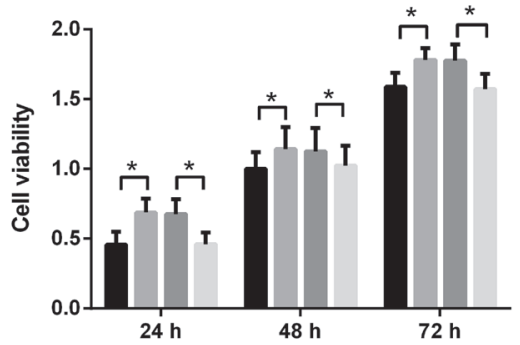

D

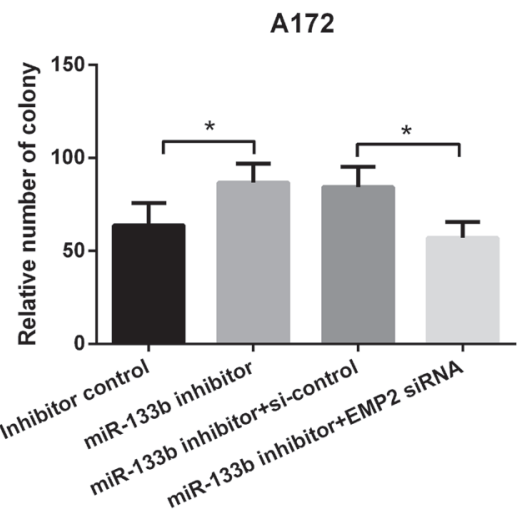

$\mathbf{F}$

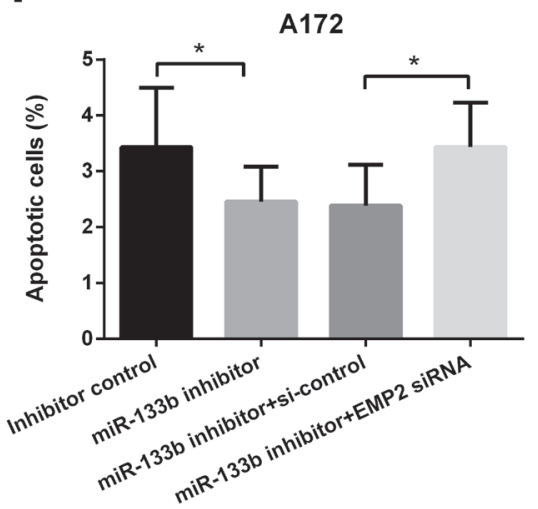

Figure 6. Inhibition of miR-133b and EMP2 expression did not promote cells viability and survival or decrease apoptosis in glioma cells in vitro. (A and B) U87 and A172 cells were transfected with miR-133b inhibitor or EMP2 siRNA. After culture for 24-72 h, cell viability was determined by MTT assay; (C and D) Proliferation of transfected U87 and A172 cells was measured by colony formation assay; (E and F) The apoptotic cells rate of transfected U87 and $\mathrm{A} 172$ cells were detected by flow cytometry. miR-133b, microRNA-133b; EMP2, epithelial membrane protein-2; siRNA, small interfering RNA; MTT, 3-(4, 5-dimethyl-2-thiazolyl)-2, 5-diphenyltetrazolium bromide; ${ }^{*}, \mathrm{p}<0.05$.

regarded as biomarkers because of their regulation effects on cell processes and their abnormal expression related to disease [23]. Recent studies indicate that some tumors, including gliomas, secrete macro-vesicles containing miRNAs [24]. Herein, we established that miR-133b has lower expres- sion in glioma than in adjacent non-tumorous tissues, and $\mathrm{miR}-133 \mathrm{~b}$ expression is therefore down-regulated in glioma.

miRNAs have important roles in a wide array of biological processes by regulating gene expression [25], and the altered miRNA expression here has promoted glioblastoma 
malignancy and contributed to proliferation, invasion, angiogenesis and multi-potency of glioma stem cells [26, 27]. Herein, miR-133b expression inhibited glioma cell biological processes in vitro, and it was effectively overexpressed or inhibited in U87 and A172 cells, respectively. This over-expression can also promote U87 and A172 cell apoptosis.

It is established that miRNAs regulate target gene expression by post-transcriptional silencing of their mRNA through complementary binding [28]. Herein, we predicted EMP2 as the miR-133b target gene in glioma, and confirmed that miR-133b regulated EMP2 expression by binding EMP2 3'UTR. EMP2 is a member of the GAS-3/PMP22 subfamily which operates as a trafficking molecule for a variety of proteins and glycolipids to efficiently transfer them from the post-Golgi endosomal compartment to the plasma membrane [29].

Further, EMP2 has recently been revealed as a novel oncogene which is up-regulated in a number of tumors such as ovarian and endometrial cancer and gliomas [30-32] and we detected up-regulated EMP2 expression in glioma. Overexpression of EMP2 enhanced cell viability and survival and inhibited apoptosis in vitro. These results confirm EMP2 is a risk factor for gliomas because the expression changes in miR-133b and its EMP2 target affect cell proliferative activity and apoptosis.

We assessed the relationship between miR-133b and glioma cell apoptosis, and our results highlighted that both miR-133b over-expression and inhibited EMP2 expression induce altered apoptosis-related factor expression. Cleavedcaspase- 3 is involved in the execution of apoptosis and can lead to programmed cell death [33] and Bax pro-apoptotic factor can antagonise Bcl-2's apoptosis inhibition [34]. Increased p38 and JNK phosphorylation levels also indicate that the apoptotic-related pathway is activated by both miR-133b over-expression and EMP2 inhibition. This establishes that miR-133b's positive regulation of glioma cell apoptosis is related to the apoptotic pathway and that miR-133b is a potential glioma suppressor.

Our experimental results establish that miR-133b inhibits cell viability and survival and promotes apoptosis in glioma cells. The process is; (1) miR-133b inhibits EMP2 expression by binding the EMP2 3'UTR and (2) this inhibition prevents the EMP2 expression which normally promotes cell viability and survival and inhibits apoptosis in vitro. Therefore, we concluded that miR-133b indirectly inhibited cell viability and survival and promoted apoptosis in glioma cells by down-regulating EMP2expression. Further, inhibition of miR-133b and EMP2 expression did not promote cell viability and survival or inhibit cell apoptosis any more in U87 and A172 cells, and this also illustrates our point. While we cannot confirm if miR-133b also acts directly on cell viability, survival and apoptosis in glioma cells, our results cannot exclude it and this possibility therefore remains prospective research.

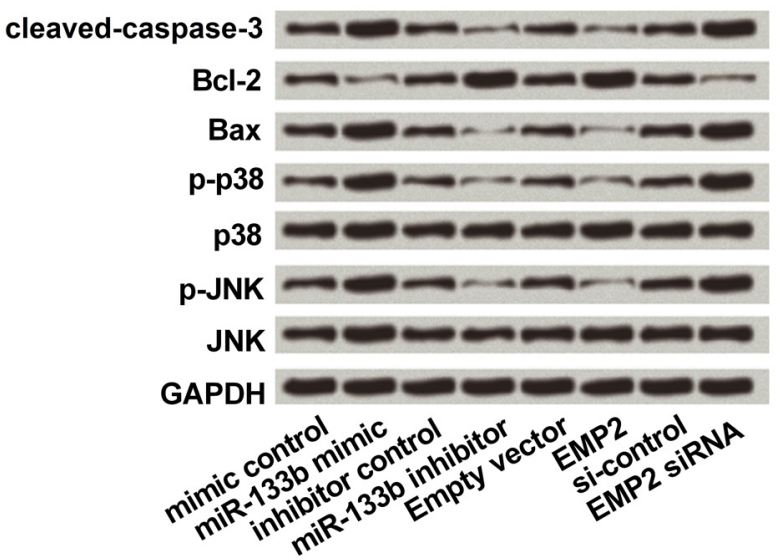

Figure 7. Over-expression of miR-133b regulated the expressions of apoptosis related factors in vitro. U87 cells were transfected with miR133b mimic, inhibitor, pcDNA3-EMP2, EMP2 siRNA or their controls, respectively. The protein immunoblots of apoptosis related factors in transfected U87 cells are shown after western blot assay. GAPDH acted as the internal control. miR-133b, microRNA-133b; EMP2, epithelial membrane protein-2; siRNA, small interfering RNA; Bcl-2, B-cell lymphoma-2; Bax, Bcl-2-associated X; p-p38, phosphorylated p38; JNK, cJun N-terminal kinase; p-JNK, phosphorylated JNK.

In conclusion, this study demonstrated that miR-133b affects cell viability, invasion and apoptosis of glioma cells by regulating the expression of EMP2 and apoptosis-related factors in vitro. This knowledge contributes to establishing a more effective method for treatment of glioma at the molecular level. Future study should elucidate miR-133b and EMP2 effects on the mechanisms involved in glioma and other cancers, and thus confirm our results.

\section{References}

[1] ZHOU Y, LIU F, XU Q, WANG X. Analysis of the expression profile of Dickkopf-1 gene in human glioma and the association with tumor malignancy. J Exp Clin Cancer Res 2010; 29 : 138. https://doi.org/10.1186/1756-9966-29-138

[2] OSTROM QT, GITTLEMAN H, LIAO P, VECCHIONEKOVAL T, WOLINSKY Y et al. CBTRUS Statistical Report: Primary brain and other central nervous system tumors diagnosed in the United States in 2010-2014. Neuro Oncol 2017; 19(suppl_5):v1-v88. https://doi.org/10.1093/neuonc/ nox158

[3] YANG H, WANG Y. Five miRNAs considered as molecular targets for predicting neuroglioma. Tumour Biol 2016; 37: 1051-1059. https://doi.org/10.1007/s13277-015-3898-9

[4] GUO YB, SHAO YM, CHEN J, XU SB, ZHANG XD et al. Effect of overexpression of HOX genes on its invasive tendency in cerebral glioma. Oncol Lett 2016; 11: 75-80. https:// doi.org/10.3892/ol.2015.3893

[5] ZHANG B, PAN X, COBB GP, ANDERSON TA. microRNAs as oncogenes and tumor suppressors. Dev Biol. 2007; 302: 1-12. https://doi.org/10.1016/j.ydbio.2006.08.028 
[6] WANG G, DAI F, YU K, JIA Z, ZHANG A et al. Resveratrol inhibits glioma cell growth via targeting oncogenic microRNAs and multiple signaling pathways. Int J Oncol 2015; 46: 1739-1747. https://doi.org/10.3892/ijo.2015.2863

[7] KANO M, SEKI N, KIKKAWA N, FUJIMURA L, HOSHINO I et al. miR-145, miR-133a and miR-133b: Tumor-suppressive miRNAs target FSCN1 in esophageal squamous cell carcinoma. Int J Cancer. 2010; 127: 2804-2814. https://doi. org/10.1002/ijc.25284

[8] HU G, CHEN D, LI X, YANG K, WANG H et al. miR-133b regulates the MET proto-oncogene and inhibits the growth of colorectal cancer cells in vitro and in vivo. Cancer Biol Ther 2010; 10: 190-197.

[9] CHEN XN, WANG KF, XU ZQ, LI SJ, LIU Q et al. MiR$133 \mathrm{~b}$ regulates bladder cancer cell proliferation and apoptosis by targeting Bcl-w and Akt1. Cancer Cell Int 2014; 14: 70. https://doi.org/10.1186/s12935-014-0070-3

[10] GABRIELY G, WURDINGER T, KESARI S, ESAU CC, BURCHARD J et al. MicroRNA 21 promotes glioma invasion by targeting matrix metalloproteinase regulators. Mol Cell Biol 2008; 28: 5369-5380. https://doi.org/10.1128/ MCB.00479-08

[11] GODLEWSKI J, NOWICKI MO, BRONISZ A, WILLIAMS S, OTSUKI A et al. Targeting of the Bmi-1 oncogene/stem cell renewal factor by microRNA-128 inhibits glioma proliferation and self-renewal. Cancer Res 2008; 68: 9125-9130. https://doi.org/10.1158/0008-5472.CAN-082629

[12] CHEN XJ, WU MY, LI DH, YOU J. Apigenin inhibits glioma cell growth through promoting microRNA-16 and suppression of BCL-2 and nuclear factor- $\mathrm{kB} / \mathrm{MMP}-9$. Molecular medicine reports. 2016; 14: 2352-2358. https://doi. org/10.3892/mmr.2016.5460

[13] LIVAK KJ, SCHMITTGEN TD. Analysis of relative gene expression data using real-time quantitative PCR and the 2(-Delta Delta C(T)) Method. Methods. 2001; 25: 402-408. https://doi.org/10.1006/meth.2001.1262

[14] NIE D, LIU R, LI J, TENG Z, ZHANG Z et al. Overexpressed MicroRNA-182 Promotes Proliferation and Invasion in Prostate Cancer PC-3 Cells by Down-Regulating N-myc Downstream Regulated Gene 1 (NDRG1). PLoS One 2013; 8: e68982. https://doi.org/10.1371/journal.pone.0068982

[15] HALANI SH, ADAMSON DC. Clinical utility of 5-aminolevulinic acid $\mathrm{HCl}$ to better visualize and more completely remove gliomas. Onco Targets Ther 2016; 9: 5629-5642. https://doi.org/10.2147/OTT.S97030

[16] MOMIYAMA M, HIROSHIMA Y, SUETSUGU A, TOME Y, MII S, YANO S et al. Enhanced resection of orthotopic red-fluorescent-protein-expressing human glioma by fluorescence-guided surgery in nude mice. Anticancer Research. 2013; 33: 11.

[17] RAMPLING R1, JAMES A, PAPANASTASSIOU V. The present and future management of malignant brain tumours: surgery, radiotherapy, chemotherapy. J Neurol Neurosurg Psychiatry 2004; 75: ii24-30. https://doi.org/10.1136/ jnnp.2004.040535
[18] FAN XZ, MAO Q, HUANG JN, ZHU SH, HU SG et al. The Influence on CD4 +CD25 +Neuropilin-1 +Treg of Peripheral Blood and the Short-term Curative Effect in Recurrent Glioma Patients Treated with Cyberknife Treatment. Chinese Journal of Neuro-Oncology. 2013. http://en.cnki. com.cn/Article_en/CJFDTotal-CJNO201302010.htm

[19] HUSE JT, HOLLAND EC. Targeting brain cancer: advances in the molecular pathology of malignant glioma and medulloblastoma. Nat Rev Cancer 2010; 10: 319-331. https://doi. org/10.1038/nrc2818

[20] LOUIS DN, OHGAKI H, WIESTLER OD, CAVENEE WK, BURGER PC et al. The 2007 WHO classification of tumours of the central nervous system. Acta Neuropathol 2007; 114: 97-109. https://doi.org/10.1007/s00401-007-0243-4

[21] WEN PY, KESARI S. Malignant gliomas in adults. N Eng J Medicine 2008; 359: 492-507. https://doi.org/10.1056/ NEJMra0708126

[22] HOELZINGER DB, DEMUTH T, BERENS ME. Autocrine factors that sustain glioma invasion and paracrine biology in the brain microenvironment. J Natl Cancer Inst 2007; 99: 1583-1593. https://doi.org/10.1093/jnci/djm187

[23] WANG W, SUN G, ZHANG L, SHI L, ZENG Y. Circulating MicroRNAs as Novel Potential Biomarkers for Early Diagnosis of Acute Stroke in Humans. J Stroke Cerebrovasc Dis 2014; 23: 2607-2613. https://doi.org/10.1016/j.jstrokecerebrovasdis.2014.06.002

[24] SKOG J, WURDINGER T, VAN RIJN S, MEIJER DH, GAINCHE L et al. Glioblastoma microvesicles transport RNA and proteins that promote tumour growth and provide diagnostic biomarkers. Nat Cell Biol 2008; 10: 1470-1476. https://doi.org/10.1038/ncb1800

[25] LINK A, BALAGUER F, SHEN Y, NAGASAKA T, LOZANO JJ et al. Fecal MicroRNAs as novel biomarkers for colon cancer screening. Cancer Epidemiol Biomarkers Prev 2010; 19: 1766-1774. https://doi.org/10.1158/1055-9965.EPI-10-0027

[26] FENG YA, LIU TE, WU Y. microRNA-182 inhibits the proliferation and migration of glioma cells through the induction of neuritin expression. Oncol Lett 2015; 10: 1197-1203. https://doi.org/10.3892/ol.2015.3365

[27] TO KK. MicroRNA: a prognostic biomarker and a possible druggable target for circumventing multidrug resistance in cancer chemotherapy. J Biomed Sci 2013; 20: 99. https://doi. org/10.1186/1423-0127-20-99

[28] KATAKOWSKI M, BULLER B, WANG X, ROGERS T, CHOPP M. Functional microRNA is transferred between glioma cells. Cancer Res 2010; 70: 8259-8263. https://doi. org/10.1158/0008-5472.CAN-10-0604

[29] FU M, RAO R, SUDHAKAR D, HOGUE CP, RUTTA Z et al. Epithelial membrane protein-2 promotes endometrial tumor formation through activation of FAK and Src. PLoS One 2011; 6: e19945. https://doi.org/10.1371/journal. pone.0019945

[30] HABEEB O, GOODGLICK L, SOSLOW RA, RAO RG, GORDON LK et al. Epithelial membrane protein-2 expression is an early predictor of endometrial cancer development. Cancer 2010; 116: 4718-4726. https://doi.org/10.1002/ cncr.25259 
[31] FU M, MARESH EL, SOSLOW RA, ALAVI M, MAH V et al. Epithelial membrane protein-2 is a novel therapeutic target in ovarian cancer. Clin Cancer Res 2010; 16: 3954-3963. https://doi.org/10.1158/1078-0432.CCR-10-0368

[32] FREIJE WA, CASTRO-VARGAS FE, FANG Z, HORVATH $\mathrm{S}$, CLOUGHESY $\mathrm{T}$ et al. Gene expression profiling of gliomas strongly predicts survival. Cancer Res 2004; 64: 65036510. https://doi.org/10.1158/0008-5472.CAN-04-0452
[33] CHOI BH, KIM W, WANG QC, KIM DC, TAN SN et al. Kinetin riboside preferentially induces apoptosis by modulating Bcl-2 family proteins and caspase-3 in cancer cells. Cancer Lett 2008; 261: 37-45. https://doi.org/10.1016/j.canlet.2007.11.014

[34] KHOR LY, MOUGHAN J, AL-SALEEM T, HAMMOND EH, VENKATESAN V et al. Bcl-2 and Bax expression predict prostate cancer outcome in men treated with androgen deprivation and radiotherapy on radiation therapy oncology group protocol 92-02. Clin Cancer Res 2007; 13: 3585-3590. https://doi.org/10.1158/1078-0432.CCR-06-2972 